\title{
Is national mental sport ability a sign of intelligence? An analysis of the top players of 12 mental sports
}

Emil O. W. Kirkegaard ${ }^{1}$

\begin{abstract}
Lists of top players for 12 different electronic sports (e-sports) or traditional mental sports were collected from a variety of sources (total $n=36 \mathrm{k}$ ). National cognitive ability/IQ was found to be a predictor of the relative representation of countries among the top players. This was particularly true when an overall mental sports score was calculated. Controlling for population size, this metric correlated $\mathrm{r}=.79$ with Lynn and Vanhanen's published national IQs. The relationship was fairly robust to controls for geographical region (coefficient $74 \%$ of the original). Furthermore, it had been suggested that IQ estimates for Nigeria were spuriously low due to the country's prominent performance on Scrabble. However, when performance across all other sports were taken into account, Nigeria's performance was what one would expect based on the previously reported mean IQs near 70.

Key words: intelligence, IQ, cognitive ability, mental sport, e-sport, Chess, Go, Poker, Scrabble, Starcraft 2, DOTA 2, League of Legends, LOL, Counter-Strike, Hearthstone, Overwatch, Super Smash Bros
\end{abstract}

\section{Introduction}

Enormous amounts of human time are spent on playing a variety of mental sports/games ${ }^{2}$, either involving a computer or not. A large amount of this time is spent playing competitive games, that is, against other players (PvP; player versus player) instead of against the computer (PvE; player versus environment). Major games with PvP gameplay usually have an in-game ranking list as well as large tournaments offering thousands or millions of US dollars in winnings. This naturally creates a focus on player ability, and the causes and correlates of it. Aside from time spent playing the games, an obvious

1 Ulster Institute for Social Research. Email: emil@emilkirkegaard.dk

2 Similar to Jensen (Jensen, 1998, p. 49ff), we define mental sport as sports where player performance is not strongly linked to physical features such as height, grip strength or maximum running speed. 
candidate for explaining differences in player skill is (general) cognitive ability/general intelligence ${ }^{3}$. Cognitive ability is known to be strongly related to a wide variety of vocational, educationing/training, social and health outcomes and has been described (in part) as a "very general mental capability that, among other things, involves the ability to reason, plan, solve problems, think abstractly, comprehend complex ideas, learn quickly and learn from experience.” (Gottfredson, 1997; Hunt, 2010; Jensen, 1998; Strenze, 2015). Since mastering a game, in particular a complex game, requires understanding the game mechanics, planning tactics/strategy and learning from experience, cognitive ability is a plausible cause of game skill.

A number of studies have examined the relationship between measures of cognitive ability and skill in a variety of games, both computerized (esports) and not (usually board games). For instance, a metaanalysis of studies of chess players and non-chess players round a mean group difference of .50 d (Sala et al., 2017). In another study, Quiroga et al (Ángeles Quiroga et al., 2015; see also Foroughi, Serraino, Parasuraman, \& Boehm-Davis, 2016) used administered 12 video games to 188 students as well as traditional standardized tests, and found that they measured nearly identical constructs (r's .93 to .96).

Given the positive relationships between game performance and cognitive ability seen between individuals, we might wonder whether national skill at mental sports also show relationships to national intelligence. Indeed, in a recent series of blogposts, Zambian biochemist Chanda Chisala (2016 etc.) argued that Nigerian Scrabble performance was much higher than one would expect based on its national IQ estimate (given as 71.2 by Lynn and Vanhanen). His interpretation is that the tests are missing real ability present in this and other African populations. His study, however, was not systematic and did not include many other mental sports. The purpose of this study was to systematically examine the relationship between national skill at mental sports and national cognitive ability.

\section{Data}

We chose to rely on counts of top players for games since ranking lists are widely available and they contain the necessary nationality data. For games, we included every esport listed at https://www.esportsearnings.com/games with at least 1,000 top players. This resulted in the inclusion of 8 games: DOTA 2 (dota2), League of Legends (lol), Counter-Strike: Global Offensive (csgo), Starcraft 2 (sc2), Counter-Strike (cs), Hearthstone (hs), Overwatch (ow), and Super Smash Bros. Melee (ssbm). Furthermore, we included major non-esports: Chess, Go, Poker and Scrabble. In each case, we found a major ranking website ${ }^{4}$ and scraped ${ }^{5}$ the contents.

The number of top players by game is shown in Table 3 .

3 We will use cognitive ability/intelligence in lieu of the more technical general cognitive ability, general mental ability, or general intelligence and in lieu of the less readable gca / gma / g. However, by this usage we refer to the general cognitive ability captured by the $g$ factor (Jensen, 1998).

4 These were: https://2700chess.com/all-fide-players, http://www.wespa.org/aardvark/cgi-bin/rating.cgi, https://www.goratings.org/en/, http://www.globalpokerindex.com/rankings/300/.

5 Automatically downloaded using a computer script. 


\begin{tabular}{|l|l|r|r|}
\hline Game & Esport & Top players & N unique countries \\
\hline sc2 & yes & 2179 & 64 \\
\hline lol & yes & 5072 & 80 \\
\hline dota2 & yes & 2219 & 71 \\
\hline csgo & yes & 9076 & 92 \\
\hline cs & yes & 2624 & 58 \\
\hline hs & yes & 1744 & 66 \\
\hline ow & yes & 2104 & 63 \\
\hline ssbm & yes & 2107 & 29 \\
\hline chess & no & 4942 & 103 \\
\hline go & no & 946 & 14 \\
\hline scrabble & no & 1321 & 48 \\
\hline poker & no & 1800 & 66 \\
\hline
\end{tabular}

Table 1: Sample sizes by game.

For predictor variables, we used the national IQs from Lynn and Vanhanen (2012), population sizes, country continents and regions from the U. N.

All statistical analyses were done in R. The player lists were scraped using R's rvest package (Wickham \& RStudio, 2016), and in one case UNIX's curl. See analysis code for details.

\section{Analyses}

\subsection{Counting players by country}

All datasets from https://www.esportsearnings.com/ were already given in country-level form. However, sometimes a few subnational units were used on the site which would have caused issues due to inconsistencies across datasets ${ }^{6}$ and missing covariate data. This was seen for sub-divisions of United Kingdom (England, Scotland, Wales, N. Ireland), China (Hong Kong, Macao), Denmark (Greenland, Faeroe Islands) and the Netherlands (Aruba etc.). In every case, the sub-divisions were merged with their mother countries using the motherland's name.

For the other datasets, they were given by player. Every dataset included some form of a national

6 For instance, of a given small unit is only available in half datasets, it would only seem to have particularly low performance in the others, insofar as one uses imputed values. 
identifier, either by stating the country or by having a flag. We converted the flags to their countries and aggregated to the country level.

\subsection{Modeling count data with rare persons}

The most straightforward approach to modeling count data is to convert it to the more common per capita form, which facilitates easy intuitive understanding and interpretation. Unfortunately, this transformation - a form of direct control for population size - results in a severe increase in the standard error of the estimate. To see this, consider a small country like Iceland (population 330k). Suppose we are interested in an event (person, in this case) that is very rare, with an average frequency of 1 in a million. This means we would expect 0.33 of them in Iceland on average. How would the sampling distribution look like for Iceland? We sampled this scenario 1e6 times. The results are given in Table 4.

\begin{tabular}{|r|r|r|}
\hline Event count & Count & Percent \\
\hline 0 & 719259 & 71.93 \\
\hline 1 & 237116 & 23.71 \\
\hline 2 & 39105 & 3.91 \\
\hline 3 & 4157 & 0.42 \\
\hline 4 & 335 & 0.03 \\
\hline 5 & 28 & 0.00 \\
\hline
\end{tabular}

Table 2: Iceland (330k population) sampling of rare person (1 in 1e6). 1 e6 samplings.

In $72 \%$ of the cases, no person was observed yielding a per capita rate of 0 . On the other hand, $24 \%$ of samples yielded a single person, yielding a per capita rate of 1 in 330k - about 3 times the true value. A further $\sim 4 \%$ of samples yielded higher counts and even more extreme per capita rates. Note that every sampling outcomes would yield a quite inaccurate estimate of the true per capita rate (1 in 1e6).

An alternative, but less intuitive, way of approaching the problem is to include population size in the regression model along with whatever predictors one is interested in. To avoid non-normality in residuals, usually log values are also employed. This approach avoids the extreme standard error for countries because the counts themselves are used, not the per capita rates. If we have a small country like Iceland, a small count is expected ( $\sim .3$ in the above), and small deviations around in (0-5) do not constitute major outliers in the modeling.

To illustrate this, we simulated fictive world-wide data (200 countries) where countries varied strongly in population size (sampled from the true country sizes with repeats) and in their per capita rate of top persons (from 1 in $1 \mathrm{e} 6$ to 1 in 1e9, normally distributed). Moreover, the per capita rate of the country was strongly caused $(r=.70$ ) by a single predictor (also normally distributed). The data were then 
modeled using 3 approaches: 1) per capita rate $\sim$ cause, 2) count $\sim$ population size + cause, 3 ) $\log 10($ count +1$) \sim \log 10$ (population size $)+$ cause. ${ }^{7}$ The model statistics were saved and analyzed. The results showed that the log count approach was the better than the per capita method in two ways. First, it produced much smaller p values and the most under the conventional thresholds, as shown in Figure 1. Though it should be said that the straight count approach was slightly superior.

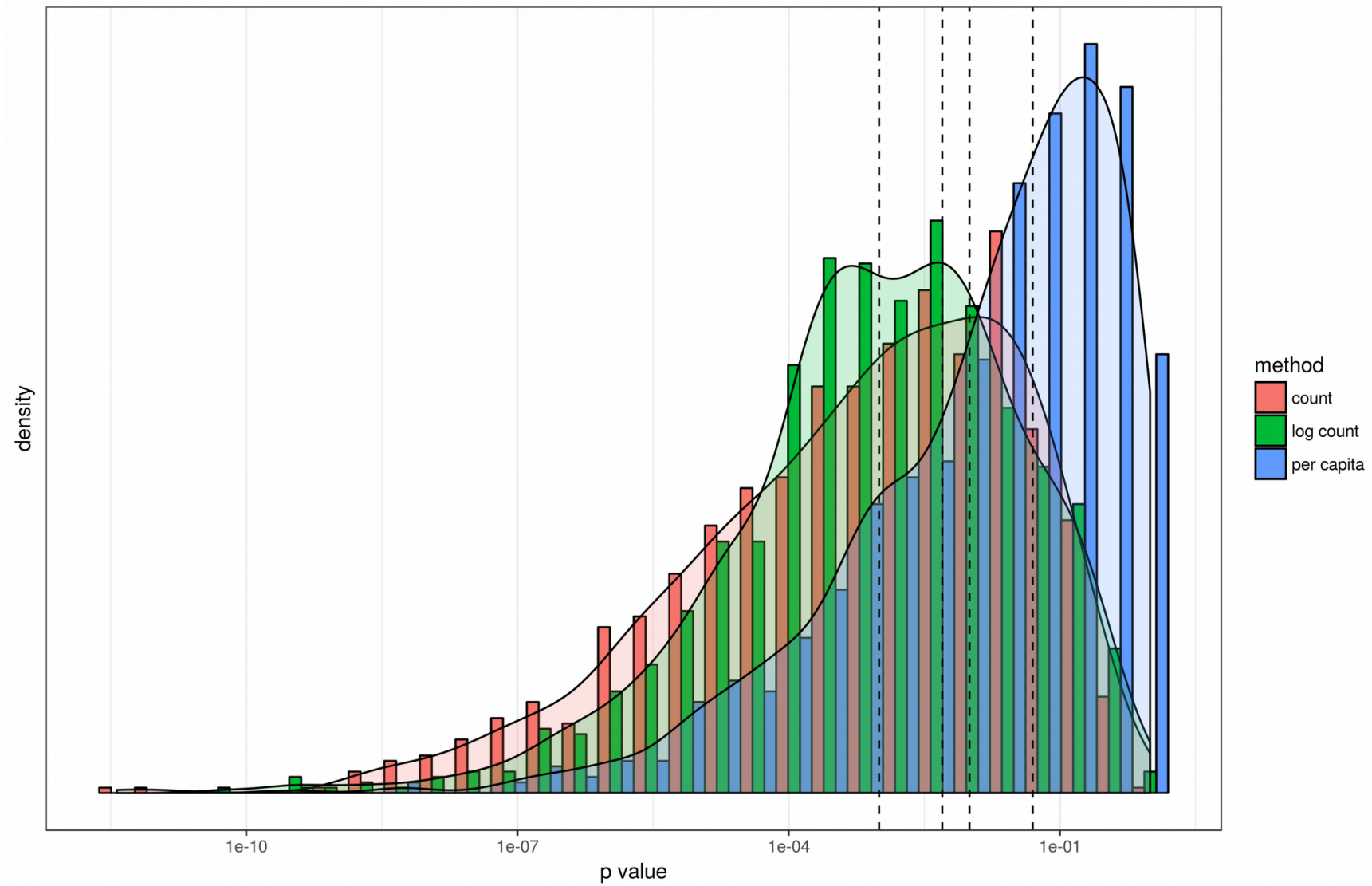

Figure 1: Distribution of $p$ values for the causal variable in simulation runs. $n=1 e 4$ runs. The vertical lines mark the traditional cutoffs of .05, .01, .005, and .001.

Second, the log count method produced the smallest variation in the beta estimates in terms of coefficient of variation (standard deviation divided by mean). Table 3 shows summary statistics for key model statistics.

\begin{tabular}{|l|l|l|l|l|l|l|l|l|l|}
\hline Method/Parameter & Mean & SD & Median & MAD & Min & Max & Skew & Kurtosis & CV \\
\hline count/beta & 3.51 & 1.78 & 3.11 & 1.37 & 0.43 & 14.08 & 1.67 & 4.01 & 0.51 \\
\hline count/p & 0.02 & 0.06 & 0.00 & 0.00 & 0.00 & 0.80 & 5.13 & 37.85 & 2.65 \\
\hline $\log$ count/beta & 0.08 & 0.03 & 0.08 & 0.02 & -0.02 & 0.18 & -0.02 & 0.16 & 0.34 \\
\hline $\log$ count/p & 0.03 & 0.09 & 0.00 & 0.00 & 0.00 & 0.98 & 5.49 & 39.27 & 2.99 \\
\hline
\end{tabular}

7 “ ” (tilde) means “is predicted by” or "regressed unto”, i.e. left-side of tilde is the dependent variable and the right hand terms are the predictors. 


\begin{tabular}{|l|l|l|l|l|l|l|l|l|l|}
\hline & & & & & & & & & \\
\hline per capita/beta & 0.13 & 0.36 & 0.12 & 0.06 & -5.21 & 7.75 & 3.63 & 269.84 & 2.84 \\
\hline per capita/p & 0.16 & 0.24 & 0.03 & 0.05 & 0.00 & 1.00 & 1.79 & 2.25 & 1.53 \\
\hline
\end{tabular}

Table 3: Summary statistics of model parameters from world simulations. $S D=$ Standard deviation, $C V$ = coefficient of variation, $M A D=$ median absolute deviation.

Note that it is not sensible to compare R2 (or adj. R2) for the modeling choices because the count approaches include population size as a predictor, and because this explains most of the variance, these models almost always have quite large R2's even when the predictor is poor.

\subsection{Predicting performance of individual mental sports}

There are a number of ways the data can be modeled. One approach is to model each dataset independently, and then meta-analyze the results. This approach will produce unbiased results insofar that sampling error is not systematic (e.g. biased towards 0 ). In every dataset, many countries had not a single top player. For these countries, 0's were imputed. After this, the following model was fit to each dataset:

$$
\log 10(\text { player count }+1) \sim \log 10(\text { population })+I Q+U N \text { macroregion }
$$

Two aggregate estimates were then computed based on the model beta estimates. The first was the arithmetic mean, which was 0.0235 . The second was a random effects meta-analytic estimate which was .0226 [.0162 to $.0289, \mathrm{p}<.0001]^{8}$. The between sample heterogeneity was $70 \%(\mathrm{p}<.0001)$, indicating substantial between sample variation unrelated to sampling error itself. This is the expected result If culture strongly affects which games populations decides to put their national talent pool towards. Figure 7 shows the forest plot.

8 95\% analytic confidence interval. The analyses was done using metafor (Viechtbauer, 2015). 


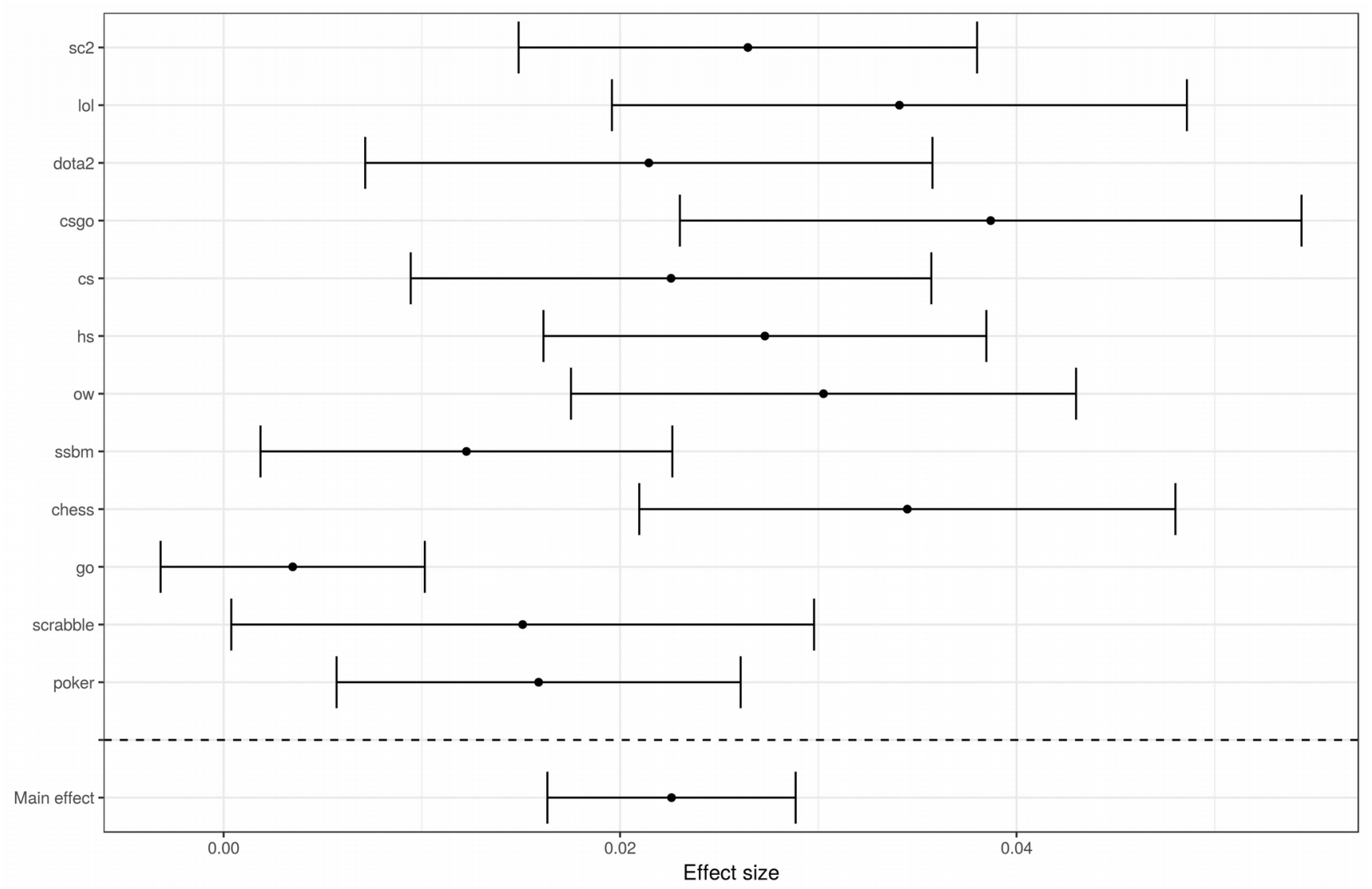

Figure 2: Forest plot for random effects meta-analysis of effect size of national IQ.

Visual inspection of the forest plot indicated that Go was an outlier, which is not surprising because it is played almost exclusively in East Asian countries (no non-East Asian country had $>=5$ players in the dataset of 946 players), producing a much larger sampling error than the analytic standard error would indicate. ${ }^{9}$ Because of this, the meta-analysis was re-run without Go. This increased the arithmetic mean effect size to .0253 and the random effects estimate to .0246 [.0195 to .0297, p < .0001].

To check robustness to analytic choices, two further modeling variants were run. First, without imputed 0's for countries with no players. Second, without the regional control. Both approaches produced higher estimates for the IQ beta at .0309 [.0229 to .0389] and .0421 [.0339 to .0504], respectively, using the random effects model.

\subsection{Aggregate mental sport performance}

An alternative approach is to avoid modeling IQ at the level of the individual game, but to aggregate game performance to a single overall metric, which is then modeled as a function of IQ and other predictors. This was done by fitting a simple population size model to each game:

$$
\log 10(\text { player count }+1) \sim \log 10 \text { (population) }
$$

9 The analytic standard error is based on the sample size and standard deviation. However, there were only 14 country observations for this dataset, the remaining values were imputed as 0 's. 
and saving the residuals. ${ }^{10}$ The residuals were then factor analyzed to see if it was sensible to interpret an aggregate metric as a measure of a latent general gaming ability. The mean inter-correlation was .57, and every game loaded positively on the general factor. Figure 8 shows the scatterplot between national IQ and general gaming ability score.

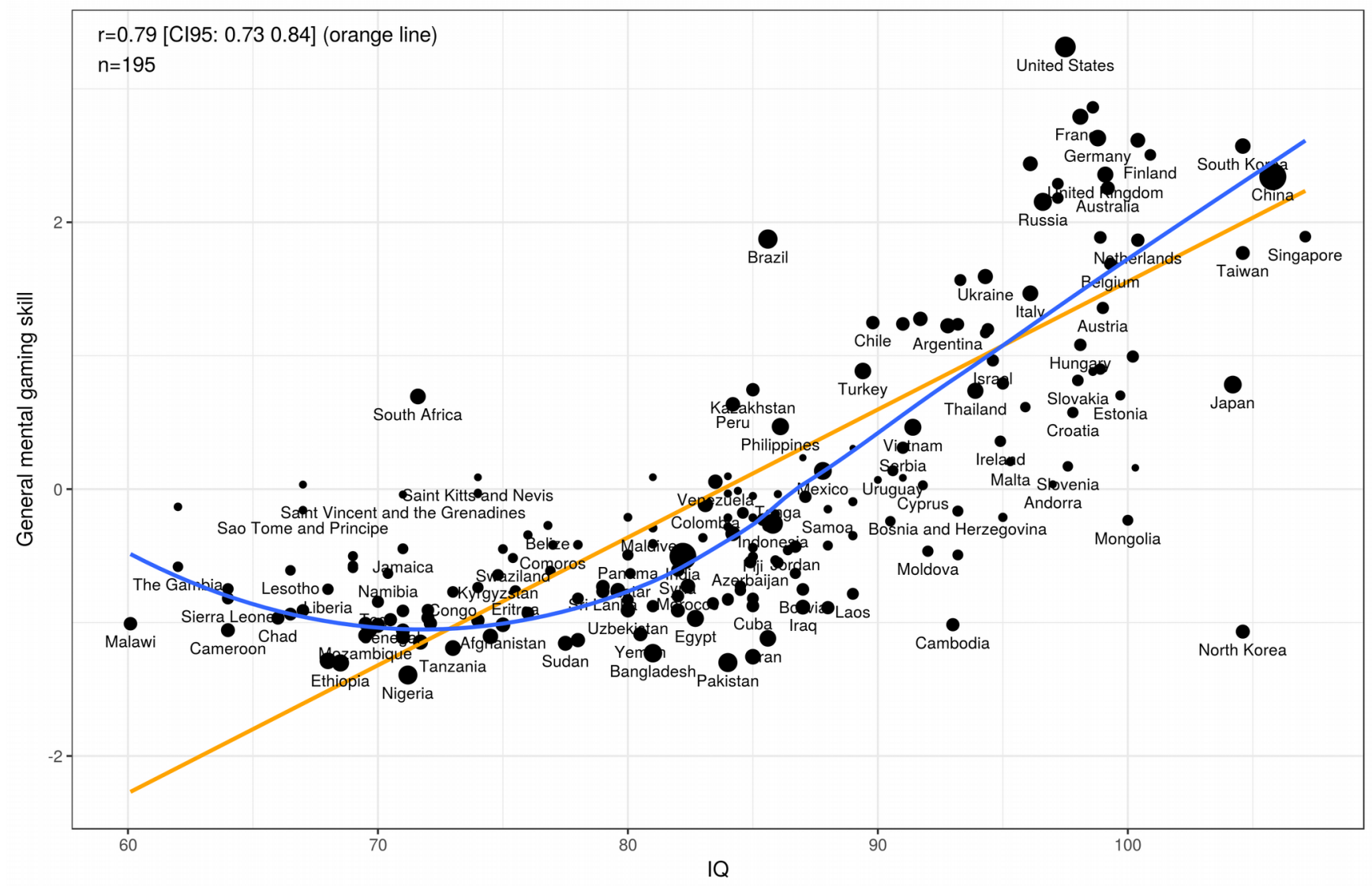

Figure 3: General gaming ability score and national IQ. Orange line = linear fit (top left), blue line = local regression fit (span $=1.00)$. Weighted by square root of population size.

The scatterplot revealed a somewhat nonlinear pattern where a large proportion of countries had similar near zero performance, roughly those below IQ 80, while there was a linear or perhaps upwardly curving trend after IQ 80. The strongest negative outlier was North Korea, presumably due to very limited internet access despite presumed high IQ. ${ }^{11}$ There were three notable positive outliers: South Africa, Brazil and United States. All of these feature a dominant European-derived population, though its fraction of the population varies, and is probably best interpreted as a smart fraction effect (La Griffe du Lion, 2002; Rindermann, Sailer, \& Thompson, 2009).

As a robustness test, the mean and median game performance were also calculated. However, these correlated

A series of regression models were fit to the final dataset. First, a simple linear regression model with

10 For this purpose, the 0-imputed datasets were used.

11 No IQ test data is available for North Korea (Lynn \& Vanhanen, 2012). However, the IQ has been estimated based on the neighboring countries (China and South Korea). 
national IQ as the predictor. Second, a nonlinear model (restricted cubic spline with 3 knots; (Harrell, 2015)) IQ to determine the gain in validity from modeling the nonlinear pattern seen in the scatterplot. The R2 adj. of this model was .693 compared to .653 of the linear model, i.e. there was a slight increase as expected ( $\mathrm{p}<5.3 \mathrm{e}-7$, for model comparison). Third, three variant models with regional controls to adjust for any culture-related confounders in mental sports interest (or interest related to the sample of included games). These were based on UN classifications of countries ( 5 continents and 23 regions), as well as a recoding of region into macro-regions to decrease the number of areas from 23 to 14 (details found in the supplementary materials). The three regional control models increased the validity beyond IQ alone and were about equivalent in terms of adj. R2 (.746, .790, .760, respectively). We used the macro-region variant for further modeling as this gave the best trade-off between likely overfitting and simplicity.

Fourth, the nonlinear term for IQ were dropped to see if the regional controls would be sufficient to account for the nonlinearity. This model had an R2 adj. of .742, only slightly worse than the nonlinear model, suggesting that cultural effects could account for most of the nonlinear IQ relationship. Still, the nonlinear model fit better and this was probably not a coincidence $(\mathrm{p}=.0001)$, and thus it was kept.

Fifth, for comparison, three models with just the regional controls were fit to see how well these could proxy for cognitive ability. While continents alone were clearly insufficient, using either regions or macro-regions produced nearly the same levels of overfit R2 adj. In case of the latter two, this is not surprising because sorting countries into 23 or 15 classes allows one to very closely approximate national IQs (R2 adj. about .89 and .90 for regions and macro-regions, respectively).

Table 4 shows the final model fit (model 2b), and Table 5 shows summary statistics for the models fitted. 


\begin{tabular}{|l|l|l|l|l|}
\hline Term & Beta & CI lower & CI upper & p \\
\hline Intercept & -0.398 & -3.841 & 3.045 & 0.820 \\
\hline IQ & 0.006 & -0.037 & 0.049 & 0.798 \\
\hline IQ' & 0.098 & 0.047 & 0.149 & $<0.001$ \\
\hline UN_macroregion=N\&W Europe + offshoots & \multicolumn{3}{|c|}{ (reference) } & \\
\hline UN_macroregion=Caribbean & -0.868 & -1.829 & 0.093 & 0.076 \\
\hline UN_macroregion=Latin America & -0.323 & -0.987 & 0.341 & 0.339 \\
\hline UN_macroregion=Central Asia & -0.697 & -1.632 & 0.237 & 0.143 \\
\hline UN_macroregion=Africa & -0.959 & -1.793 & -0.125 & 0.025 \\
\hline UN_macroregion=Eastern Asia & -1.597 & -2.151 & -1.043 & $<0.001$ \\
\hline UN_macroregion=Eastern Europe & -0.223 & -0.728 & 0.282 & 0.384 \\
\hline UN_macroregion=Melanesia & -1.005 & -2.299 & 0.290 & 0.128 \\
\hline UN_macroregion=Micronesia & -0.390 & -2.973 & 2.193 & 0.766 \\
\hline UN_macroregion=MENA & -0.999 & -1.679 & -0.319 & 0.004 \\
\hline UN_macroregion=Polynesia & -0.789 & -3.642 & 2.065 & 0.586 \\
\hline UN_macroregion=South-Eastern Asia & -0.921 & -1.510 & -0.332 & 0.002 \\
\hline UN_macroregion=Southern Asia & -1.898 & -0.431 & 0.002 \\
\hline UN_macroregion=Southern Europe & -1.224 & -0.082 & 0.025 \\
\hline
\end{tabular}

Table 4: Full model fit. $n=195$ countries. $R 2$ adj. $=.760$. Weighted by square root of population size. 


\begin{tabular}{|l|l|l|r|}
\hline Model & IQ & Regional control & Adj. R2 \\
\hline 1a & linear & no & 0.626 \\
\hline 1b & nonlinear & no & 0.676 \\
\hline 2a & nonlinear & continent & 0.790 \\
\hline 2b & nonlinear & macro-region & 0.760 \\
\hline 2c & nonlinear & region & 0.746 \\
\hline 2d & linear & macro-region & 0.742 \\
\hline 3a & no & continent & 0.386 \\
\hline 3b & no & macro-region & 0.707 \\
\hline 3c & no & region & 0.744 \\
\hline
\end{tabular}

Table 5: Summary statistics for fitted models. Weighted by square root of population size.

It is noteworthy that every regional dummy has a negative beta estimate compared to the north and west European reference group. Interpretation of this is unclear. It might reflect bias in data collection where games that non-Europeans play were missed. This seems unlikely given the inclusiveness of the dataset and the systematic sampling approach (i.e. include all games with $>=1,000$ top players). Alternatively, it might reflect a genuine cultural effect where N\&W European cultures place more emphasis on performance in mental sports, perhaps mediated through individualism.

The effect of national IQ stayed quite substantial in all models, and only partially shrunk when regional controls were added. If we compare the linear models $1 \mathrm{a}$ and $2 \mathrm{~d}$, we find that the betas for IQ were 0.095 and 0.074 , meaning that IQ retained $74 \%$ of its validity.

Finally, because we have 12 indicators of general mental sport skill, it is possible to conduct a moderator analysis using Jensen's method (method of correlated vectors), shown in Figure 4. 


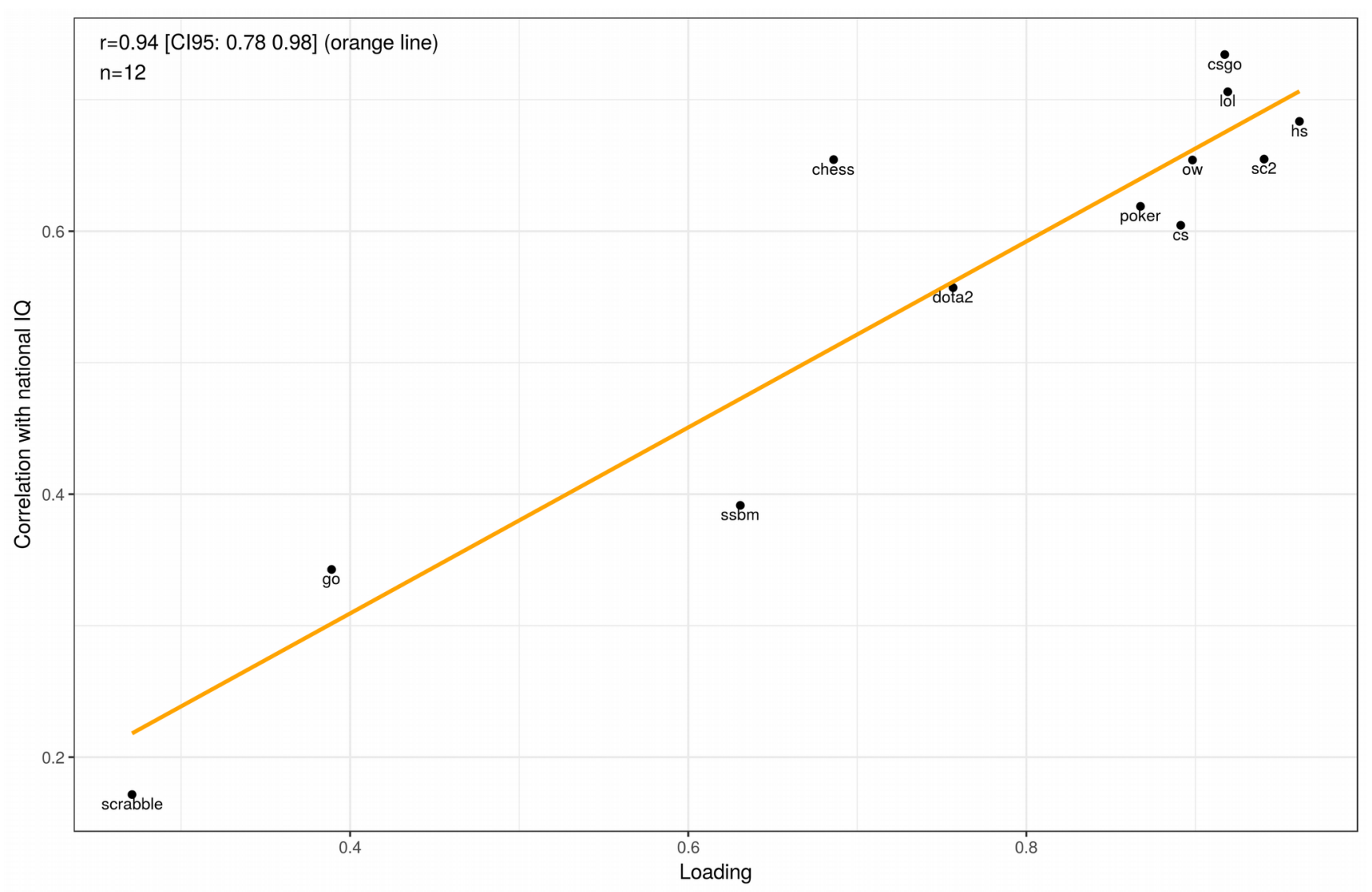

Figure 4: Jensen's method applied to the general mental sports factor.

A strong pattern is seen such that the games that more strongly reflect general gaming ability also show stronger correlations to national IQ, as predicted from a latent factor model. It also offers an explanation of the strong performance of Nigerians on Scrabble, as Scrabble was the worst indicator of general mental sports ability (factor loading $=.27$ ).

\section{Discussion and conclusion}

The present study investigated national mental gaming skill. Across 12 diverse games, it was found that skill at one game relates positively to skill at another game, supporting a general mental sports ability interpretation. Scoring the countries on this general ability results in a correlation of .79 with Lynn and Vanhanen's national IQs (weighted by square root of population size). The strong relationship between national IQ mostly held when regional controls were included. The observed decrease of about $26 \%$ validity, might reflect some cultural confounding or measurement error in the national IQs (Westfall \& Yarkoni, 2016). Nevertheless, the strong relationship fits well with both individual level results obtained in multiple studies (Ángeles Quiroga et al., 2015; Foroughi et al., 2016; Kokkinakis, Cowling, Drachen, \& Wade, 2017; Spitz, 1978), as well as large between country differences in cognitive ability reported by a number of authors (Lynn \& Vanhanen, 2012; Rindermann, 2007).

With regards to the arguments by Chisala concerning Nigeria and Scrabble, we find no support for mismeasured Nigerian intelligence. It is true, as he noted, that Nigerians are much better at Scrabble 
than one would expect (standardized residual for Scrabble is 1.55, rank 3). However, Nigerians underperform on the remaining 11 sports (all residuals are negative), and the overall factor score of Nigeria (-1.39) is about what one would expect based on the current estimates of the country's mean cognitive ability and the relation to national IQ. Indeed, based on a linear regression with just IQ, the standardized residual for Nigeria is only -0.16 , meaning that the country performs very slightly worse at mental sports in general than one would expect based on its mean national intelligence. The predicted IQ of Nigeria was 73.1 based on a nonlinear model with just game performance as the predictor, Lynn and Vanhanen's (2012) estimate was 71.2, and a recent large-scale study (n $\approx 11 \mathrm{k}$ ) using Raven's Standard Progressive Matrices found a mean IQ of 65.5 (Hur, Nijenhuis, \& Jeong, 2017). ${ }^{12}$ The anomalously high Scrabble performance is not plausibly interpreted as hidden ability, but rather as a cultural specific preference for a specific sport.

\section{Supplementary material and acknowledgments}

Supplementary materials including code, high quality figures and data can be found at https://osf.io/7xpeh/.

\section{References}

Ángeles Quiroga, M., Escorial, S., Román, F. J., Morillo, D., Jarabo, A., Privado, J., ... Colom, R. (2015). Can we reliably measure the general factor of intelligence (g) through commercial video games? Yes, we can! Intelligence, 53(Supplement C), 1-7.

https://doi.org/10.1016/j.intell.2015.08.004

Becker, D. (2017, November 30). THE NIQ-DATASET. Retrieved from http://viewoniq.org/? page_id $=9$

Chisala, C. (2016, October 31). Scrabble Spells Doom for the Racial Hypothesis of Intelligence. Retrieved from http://www.unz.com/article/scrabble-spells-doom-for-the-racial-hypothesis-ofintelligence/

Foroughi, C. K., Serraino, C., Parasuraman, R., \& Boehm-Davis, D. A. (2016). Can we create a measure of fluid intelligence using Puzzle Creator within Portal 2? Intelligence, 56(Supplement C), 58-64. https://doi.org/10.1016/j.intell.2016.02.011

Gottfredson, L. S. (1997). Mainstream science on intelligence: An editorial with 52 signatories, history, and bibliography. Intelligence, 24(1), 13-23.

12 This study did not calculate a norm referenced IQ score. However, the conversion is provided by David Becker's National IQ dataset, v1.2 (Becker, 2017). 
Harrell, F. E. (2015). Regression Modeling Strategies: With Applications to Linear Models, Logistic and Ordinal Regression, and Survival Analysis (2nd ed. 2015 edition). New York: Springer.

Hunt, E. (2010). Human Intelligence. Cambridge University Press.

Hur, Y.-M., Nijenhuis, J. te, \& Jeong, H.-U. (2017). Testing Lynn’s Theory of Sex Differences in Intelligence in a Large Sample of Nigerian School-Aged Children and Adolescents $(\mathrm{N}>11,000)$ using Raven’s Standard Progressive Matrices Plus. Mankind Quarterly, 57(3). Retrieved from http://mankindquarterly.org/archive/issue/57-3/11

Jensen, A. R. (1998). The g factor: the science of mental ability. Westport, Conn.: Praeger.

Kokkinakis, A. V., Cowling, P. I., Drachen, A., \& Wade, A. R. (2017). Exploring the relationship between video game expertise and fluid intelligence. PLOS ONE, 12(11), e0186621. https://doi.org/10.1371/journal.pone.0186621

La Griffe du Lion. (2002, March 1). The smart fraction theory of IQ and the wealth of nations. Retrieved from http://www.lagriffedulion.f2s.com/sft.htm

Lynn, R., \& Vanhanen, T. (2012). Intelligence: A Unifying Construct for the Social Sciences (1st ed.). Ulster Institute for Social Research.

Rindermann, H. (2007). The g-factor of international cognitive ability comparisons: the homogeneity of results in PISA, TIMSS, PIRLS and IQ-tests across nations. European Journal of Personality, 21(5), 667-706. https://doi.org/10.1002/per.634

Rindermann, H., Sailer, M., \& Thompson, J. (2009). The impact of smart fractions, cognitive ability of politicians and average competence of peoples on social development. Talent Development \& Excellence, 1(1). Retrieved from http://www.iratde.org/issues/1-2009/tde_issue_12009_03_rindermann_et_al.pdf

Sala, G., Burgoyne, A. P., Macnamara, B. N., Hambrick, D. Z., Campitelli, G., \& Gobet, F. (2017). Checking the “Academic Selection” argument. Chess players outperform non-chess players in cognitive skills related to intelligence: A meta-analysis. Intelligence, 61, 130-139. https://doi.org/10.1016/j.intell.2017.01.013

Spitz, H. H. (1978). The universal nature of human intelligence: Evidence from games. Intelligence, 
2(4), 371-379. https://doi.org/10.1016/0160-2896(78)90026-0

Strenze, T. (2015). Intelligence and Success. In S. Goldstein, D. Princiotta, \& J. A. Naglieri (Eds.), Handbook of Intelligence (pp. 405-413). New York, NY: Springer New York. Retrieved from http://link.springer.com/10.1007/978-1-4939-1562-0_25

Viechtbauer, W. (2015). metafor: Meta-Analysis Package for R (Version 1.9-8). Retrieved from https://cran.r-project.org/web/packages/metafor/index.html

Westfall, J., \& Yarkoni, T. (2016). Statistically Controlling for Confounding Constructs Is Harder than You Think. PLOS ONE, 11(3), e0152719. https://doi.org/10.1371/journal.pone.0152719

Wickham, H., \& RStudio. (2016). rvest: Easily Harvest (Scrape) Web Pages (Version 0.3.2). Retrieved from https://cran.r-project.org/web/packages/rvest/index.html 